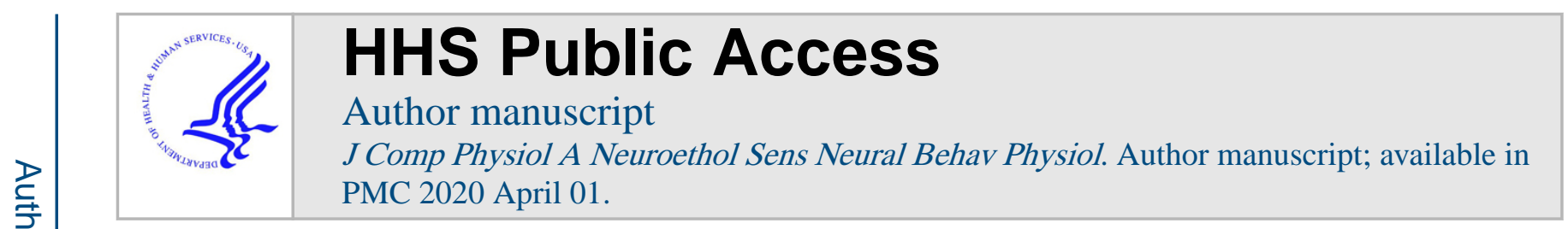

Published in final edited form as: J Comp Physiol A Neuroethol Sens Neural Behav Physiol. 2019 April ; 205(2): 191-202. doi:10.1007/ s00359-019-01317-5.

\title{
Behavioral Analysis of Substrate Texture Preference in a Leech, Helobdella austinensis
}

\author{
Rachel C. Kim ${ }^{1}$, Dylan Le ${ }^{2}$, Kenny Ma ${ }^{1}$, Elizabeth A. C. Heath-Heckman ${ }^{1,3}$, Nathan \\ Whitehorn ${ }^{4}$, William B. Kristan Jr. ${ }^{2}$, and David A. Weisblat ${ }^{1, *}$ \\ ${ }^{1}$ Department of Molecular and Cell Biology, 385 LSA, University of California, Berkeley, CA \\ 94720-3200 \\ ${ }^{2}$ Division of Biological Sciences, 3119 Pacific Hall, University of California San Diego, La Jolla, \\ CA 92093 \\ ${ }^{3}$ Present Address: Department of Ecology and Evolutionary Biology, University of California, Los \\ Angeles, CA \\ ${ }^{4}$ Department of Physics and Astronomy, University of California, Los Angeles, CA
}

\section{Abstract}

Leeches in the wild are often found on smooth surfaces, such as vegetation, smooth rocks or human artifacts such as bottles and cans, thus exhibiting what appears to be a "substrate texture preference". Here, we have reproduced this behavior under controlled circumstances, by allowing leeches to step about freely on a range of silicon carbide substrates (sandpaper). To begin to understand the neural mechanisms underlying this texture preference behavior, we have determined relevant parameters of leech behavior both on uniform substrates of varying textures, and in a behavior choice paradigm in which the leech is confronted with a choice between rougher and smoother substrate textures at each step. We tested two non-exclusive mechanisms which could produce substrate texture preference: 1) a Differential Diffusion mechanism, in which a leech is more likely to stop moving on a smooth surface than on a rough one, and;2) a Smoothness Selection mechanism, in which a leech is more likely to attach its front sucker (prerequisite for taking a step) to a smooth surface than to a rough one. We propose that both mechanisms contribute to the texture preference exhibited by leeches.

\section{Keywords}

Helobdella; leech; neuroethology; texture discrimination; touch-mediated behavior

*Corresponding author: weisblat@berkeley.edu; 510-642-8309; ORCID http://orcid.org/0000-0002-9617-4130. ETHICAL APPROVAL

This article does not contain any studies with human participants or vertebrate animals performed by any of the authors. 


\section{INTRODUCTION}

Although every cell will respond to mechanical stimuli at some intensity level (Belas 2014), animal nervous systems contain specialized mechanosensory neurons that mediate a tremendous diversity of behavioral responses to a wide range of mechanical stimuli (Kocer 2015). The relative simplicity of invertebrate systems makes them useful for identifying mechanosensory transducers and the behaviors they mediate. For example, studies in several leech species (Hirudo medicinalis, H. verbana, Macrobdella decora, Haementeria ghilianii, Erpobdella obscura) suggest that all leeches have three classes of segmentally iterated mechanosensory cells: three pairs of T cells, which respond to light touch; two pairs of $\mathrm{P}$ cells, which respond to moderate pressure, and two pairs of $\mathrm{N}$ cells, multimodal nociceptors that respond to acid, heat, and intense mechanical inputs such as pinching (Nicholls and Baylor 1968; Kramer and Kuwada 1983; Kuwada and Kramer 1983; Nusbaum and Kristan 1986; Pastor et al. 1996; Baltzley et al. 2010). In addition to their different functional ranges of mechanical activation, $\mathrm{T}$ and $\mathrm{P}$ neurons differ in their dynamics: $\mathrm{T}$ cells respond best to transient or moving stimuli whereas P cells prefer sustained stimulation (Nicholls and Baylor 1968; Carlton and McVean 1995; Lewis and Kristan 1998b). Activation of T and P cells in different body locations (front, middle, and back) elicits different responses: shortening, local bending, and locomotion, both crawling and swimming (Kristan 1982; Kristan et al. 2005; Palmer et al. 2014).

At the behavioral level, the reflexive bending of the medicinal leech body wall in response to $P$ cell stimulation has been used to reveal how location and intensity of mechanical stimuli are encoded by population coding vectors (Lewis and Kristan, 1998a 1998b). In addition, the initiation and modulation of shortening and swimming behaviors by mechanosensory inputs has been documented (Shaw and Kristan 1997; Kristan et al. 2005). Behavioral comparisons across leech species have revealed intriguing differences as well. For example, activation of P cells in the midbody of $H$. verbana produces a local bending response away from the site of stimulation whereas the same $\mathrm{P}$ cell activation in E. obscura causes them to roll themselves into a tight coil (Baltzley et al. 2010). In addition, activation of $\mathrm{P}$ or $\mathrm{N}$ cells in the back end of most leech species normally initiates locomotor behavior (crawling or swimming) (Kristan 1982; Palmer et al. 2014) but these responses are inhibited by feeding behavior specifically among various sanguivorous (blood-sucking) species, for which feeding opportunities are rare and of high energetic value, but not in predaceous species, for which feeding opportunities are more common and less valuable energetically (Gaudry et al. 2010).

Another leech behavior that would seem to be dependent on mechanosensation is suggested by the common knowledge (among leech gatherers at least) that submerged bottles, cans, and smooth rocks are excellent places to find leeches. At first glance, this behavior, which we term substrate texture selection, appears to involve a "preference" for smoother surfaces compared to rougher ones, but other explanations are possible. For example, it might be easier to detect and/or collect the animals from smooth objects. Moreover, even if the leeches do accumulate preferentially on smooth surfaces, this might involve a comparison of mechanosensory inputs by the animal or simply a "diffusion trap" in which leeches crawl 
longer or more rapidly on rough surfaces than on smooth surfaces. Here, we first demonstrate the existence of substrate texture preference under controlled conditions and then examine its behavioral basis, using a small glossiphoniid leech, Helobdella austinensis (Kutschera et al. 2013).

\section{MATERIALS AND METHODS}

\section{Animals}

Adult leeches (Helobdella austinensis; Kutschera et al., 2013) were obtained from a laboratory breeding colony kept at room temperature (ca. $21-25^{\circ} \mathrm{C}$ ) in artificial pond water (1\% artificial seawater [Salinity for Reefs; AquaVitro]. Animals were housed in freely breeding groups of mixed ages, ranging from a few dozen to a few hundred individuals in 0.5-2 liter glass or plastic containers. Animals were fed 2-5 times per week by adding several grams of frozen midge larvae (BloodWorms; Hikari or Omega One) to each bowl and then changing the water after the animals had fed to satiety (2-5 hours). The state of satiety was readily judged by the extent to which their guts--easily visualized through the translucent body wall--were filled with red pigment from the ingested midge larvae.

The animals used were all free-living adults. Variations in size reflected a combination of age and prior success in feeding. Individual animals to be tested were weighed before use on an analytical balance, by quickly blotting them dry and then placing them into a small container of pond water for which the tare weight had already been determined.

\section{Split Dish Substrate Texture Preference Assay}

Leeches were given at least 2 hours to feed before each trial. After feeding, individuals to be used were blotted dry and weighed using an analytical scale. Animals bearing embryos or juveniles were not used.

Assay chambers consisted of circular plastic petri dishes (Falcon; 60mm diameter, $15 \mathrm{~mm}$ depth), the internal surfaces (bottom and sides) of which were lined with waterproof silicon carbide sandpaper (3M Wetordry $\left.{ }^{\mathrm{TM}}\right)$, which was glued to the plastic surface by either vinyl or spray contact adhesive (3M). The two halves of each chamber were lined with different grades of sandpaper, ranging from 80 to 1500 grit, respectively. Each dish was filled with $13 \mathrm{~mL}$ of spring water to a depth of $5 \mathrm{~mm}$ and placed inside a light-proof container illuminated from above with infrared (IR) LEDs. Between each trial, dishes were rinsed thoroughly with deionized water. For each assay, one leech was placed in the center of the rougher grade sandpaper at the beginning of the trial. Each trial lasted 25 minutes, and started once the light-tight container was placed over the dish. The trial duration of 25 minutes was selected as an interval after which most animals had ceased moving following the arousal caused by their introduction into the experimental chamber. Trials in which an individual failed to approach the smoother substrate were discarded. A new leech was used for each trial. All trials were carried out at room temperature (RT; roughly $22^{\circ} \mathrm{C}$ ).

Images were acquired using a D-Link Wi-Fi camera (1 MPixel) or a Samsung Galaxy S4 Zoom camera (16 Mpixels; modified by removing the factory-installed IR filter) supported 
inside the light-proof container, above the dish and level with the IR LED illumination. Pictures were taken once every 10 seconds for 25 minutes (150 frames per trial). The illumination we used produced no measurable increase in water temperature.

Data analysis. For each trial, the time spent on each side was calculated, with the position at each time point defined by the location of the posterior sucker. Because pictures were taken once every ten seconds, which was short relative to the average step frequency, it was assumed that there was at most one step taken within the ten seconds following each picture. Percentage of time spent on each grade was calculated, and deviation from random preference (50\% of time spent on each grade) was assessed by a t-test with a post-hoc Bonferroni correction for multiple comparisons.

\section{Checkerboard Substrate Texture Preference Assay}

Leeches were prepared and selected according to the protocol described above. Assay chambers consisted of square plastic petri dishes (Fisherbrand; $100 \mathrm{~mm} \times 100 \mathrm{~mm}$ ), the dishes were lined with waterproof silicon carbide sandpaper (3M Wetordry ${ }^{\mathrm{TM}}$ ), as described above, except that the bottoms of the dishes were lined with a $6 \times 6$ checkerboard pattern with alternating squares $(13 \mathrm{~mm} \times 13 \mathrm{~mm})$ of two selected textures. In each checkerboard chamber, the sides were lined with the rougher of the two textures under comparison, to discourage the leech from attaching to the sides of the chamber. Each dish was filled with 35 $\mathrm{mL}$ of spring water to a depth of $5 \mathrm{~mm}$, and placed in the same light-proof container as above. For each comparison (e.g. 80 grit vs 1500 grit squares), two series of trials were conducted, started by placing a leech on a rough square and on a smooth square, respectively. Between each trial, chambers were rinsed thoroughly with deionized water. Assays began with one leech placed on a center rough or smooth square under the light-tight container, and ended after 5 minutes. A new leech was used for each trial. All trials were carried out at RT, roughly $22^{\circ} \mathrm{C}$. Videos were acquired according to the same protocol as above.

Data analysis.-From all the steps taken in each set of trials, we used a Monte Carlo simulation approach to estimate the probabilities of the four possible transitions, i.e., from smooth to rough, from smooth to smooth, from rough to smooth and from rough to rough-$\mathrm{p}(\mathrm{SR}), \mathrm{p}(\mathrm{SS}), \mathrm{p}(\mathrm{RS})$ and $\mathrm{p}(\mathrm{RR})--$ and to test whether the data were consistent with leeches making the four transitions randomly. For this purpose, in each trial, each step taken was sorted into one of four categories, based on the substrate texture at the origin and the termination of the step: from smooth to smooth, from smooth to rough, from rough to rough and from rough to smooth--this corresponded to four categories of transitions, abbreviated SS, SR, RR and RS, respectively. For each experiment, we evaluated the preferences of the leeches for each of the four possible transitions by comparing the total number of transitions in each category to the numbers predicted by a Monte Carlo simulation modeling the behavior of an identical number of simulated leeches, with the same starting conditions (i.e., starting on the rough or smooth surface), and the same number of transitions (steps) for each leech. In our Monte Carlo simulations, we assumed a memoryless process with fixed, time- 
independent probabilities for each type of transition: $p(R S), p(R R)=1-p(R S), p(S R)$, and $\mathrm{p}(\mathrm{SS})=1-\mathrm{p}(\mathrm{SR})$.

We then found the values of these probabilities that best match the data. For each experiment, we defined "best match" by an objective function equal to the sum of the squares of the differences in each of the four total transition counts between a simulation with a given set of transition probabilities and the observed data; in the limit of Gaussian errors, this provided an optimal maximum-likelihood estimator (Neyman and Pearson, 1933). We estimated these expected values by taking the means of the transition counts from 50 simulations run for each set of transition probabilities in a grid with test points corresponding to differences in probability of 0.01 .

To calculate $p$-values, we first ran simulations of the parameters $\mathrm{p}(\mathrm{RS})=\mathrm{p}(\mathrm{SR})=0.5$, corresponding to the leeches moving randomly between the two substrate textures. For each simulation, we tabulated the values of the objective function tested against the $\mathrm{p}(\mathrm{RS})=$ $\mathrm{p}(\mathrm{SR})=0.5$ hypothesis, which generated the statistical distribution of how well the behavioral data matched expectations by chance. We then counted the fraction of these simulation runs that, by chance, fit $\mathrm{p}(\mathrm{RS})=\mathrm{p}(\mathrm{SR})=0.5$ as well as or more poorly than the data to give the final quoted $p$ values. The programs written to carry out these analyses are provided as Supplemental File 1.

Uniform Substrate Texture Response Analysis-Leeches to be used in these studies were anesthetized by immersion in cold water $\left(5^{\circ} \mathrm{C}\right)$ to allow for removal of any attached eggs and juvenile leeches, then maintained individually in $9 \times 50 \mathrm{~mm}$ plastic petri dishes filled with artificial pond water for at least 24 hours at room temperature before testing. Animals were fed on bloodworms (Omega One) 24 hours prior to experimentation.

Step frequency assay.-Assay chambers (Falcon $9 \times 50 \mathrm{~mm}$ plastic petri dishes) were constructed as described above except that their insides were covered with a single grade of silicon carbide (3M Wetordry ${ }^{\mathrm{TM}}$ ) sandpaper (80, 150, 320, 400, 600, or 1500 grit); unlined petri dishes served as controls. Each leech was placed in a pond water-filled chamber and observed for 30 minutes after its first complete step. Experiments were initially conducted in red light (Fostec 8375 light source through Bleyer red gift wrapping paper), because leeches are insensitive to red light (Jellies 2014). We also compared Helobdella in white light and found that their stepping behavior on different sandpaper grits was statistically indistinguishable from those tested in red light. Thus, for convenience, later trials were run exclusively in white light. Each leech was tested on every grade of sandpaper, in a random order, then kept for at least 30 minutes in an unlined petri dish before being transferred onto another grade of sandpaper for the next testing session.

Head withdrawal assay.-Using the same chambers, we counted the number of times the animal lifted its anterior sucker from the surface of the sandpaper before re-attaching this sucker to complete a step. Viewed from above (i.e., the dorsal surface of the leech), head raises were distinct from other movements of the anterior region because the ventrallylocated anterior sucker became visible. Head raises accompanied by rapid shortening of the 
body were classified as head withdrawals. These experiments were conducted exclusively in red light, and animals were given 30 minutes of recovery time in plastic petri dishes before being placed into another sandpaper chamber. Animals were recorded using a Point Grey Flea3 USB camera mounted on the trinocular head of a dissecting microscope (15 fps).

\section{RESULTS}

\section{Heavy but not light $\boldsymbol{H}$. austinensis accumulate preferentially on smoother surfaces.}

The fact that, in the wild, leeches are often found on the undersides of submerged objects such as cans, bottles, vegetation, or smooth rocks (Adamiak-Brud et al. 2015; personal observations) led us to ask if leeches have the ability to distinguish between different textures, as judged by the ability to reliably exhibit a preference for one texture over another under controlled conditions.

Leeches in the genus Hirudo exhibit three distinct modes of locomotion: swimming (Kristan et al. 2005), vermiform crawling (as seen in earthworms), or an inchworm-like stepping (Stern-Tomlinson et al. 1986). In contrast to Hirudo, leeches in the genus Helobdella seem unable to swim and seldom exhibit vermiform crawling. Instead, they rely primarily on the inchworm-like stepping mode of locomotion. Each definitive step is often preceded by a side-to-side scanning behavior of the anterior end, as if it were sampling the surrounding area before taking a step. Similar scanning behavior has been documented for the European medicinal leech, Hirudo verbana (Harley and Wagenaar 2014).

To determine if leeches exhibit a substrate texture preference, we observed the behavior of individual $H$. austinensis in dishes lined with rough and smooth sandpaper (Fig. 1). From many such trials, we calculated the percentage of time spent on each texture.

In the first series of experiments, we tested leeches on a fixed comparison of 600 grit (finer) versus 100 grit (coarser) sandpaper. These experiments revealed a weak preference for the finer grit (Fig. 1c). Closer analysis revealed that lighter leeches (in the weight range of 2-20 $\mathrm{mg}$ ) showed no clear preferences, but that the heavier leeches (20-35 mg) exhibited a significant preference for the smoother substrate $(p<0.001)$ (Fig. 1d). One possible explanation for this preference is that smaller, lighter individuals do not experience a force from the substrate that is sufficient to activate appropriate mechanoreceptors, whereas larger, heavier individuals do. Alternatively, it could be that the surface area of the sucker somehow impacts the animal's perception of the substrate texture or its ability to establish a firm grip. Whatever the cause, based on their more reliable substrate texture response, only leeches weighing more than $20 \mathrm{mg}$ were used for further experiments.

\section{Leeches may discriminate across a range of substrate textures.}

To assess the leeches' ability to discriminate between different substrate textures, we used the same split dish assay to test for substrate preferences in pairwise comparisons of various textures ranging from coarse ( 80 grit) to fine (1500 grit). In these experiments we compared each extreme of the texture range against all other values, i.e., 80 vs $150,220,320,600$, and 1500 grit, as well as 1500 vs $150,220,320$, and 600 grit. As expected, the smoother surface 
was favored in all assays that revealed a significant substrate texture preference, but the ability of the leeches to discriminate textures was limited (Fig. 1e). In this experimental paradigm, only leeches confronting the more dramatic differences ( 80 vs 1500 and 80 vs 600) exhibited a statistically significant preference for smooth surfaces, but there is an apparent trend for preferring the smoother surface in most comparisons. Leeches did spend significantly more time (an average of $71.79 \%$ ) on the 600 grit than on the 80 grit sandpaper $(p<0.05$ ), and even more time on the 1500 grit (an average of $79.81 \% p<0.001$ ) rather than the 80 grit paper. Conversely, in experiments where the 1500 grit surface was compared against all other surfaces, the leeches preferred the smoother surface on average, but the preference was statistically significant only for 1500 vs 80 grit (Fig. 1f).

These results demonstrate that leeches do accumulate on the smoother of two surfaces, at least when there is a large difference in the texture of the substrate. To test how leeches end up on the smoother surface in the bisected smooth/rough petri dish, we considered two general classes of behavioral possibilities. First, given that animal dispersal is often modeled mathematically as a diffusional process (Blackwell 1999; Horen et al. 2007; Smouse et al. 2010), we considered a strategy of Differential Diffusion, in which the leech steps in random directions ("diffusion"), but moves more slowly on smoother surfaces, analogous to a smaller coefficient of diffusion. This would lead to individuals accumulating on smoother surfaces. Second, we considered Smoothness Selection strategies, in which the leech preferentially moves onto smoother surface when confronted with a border between rough and smooth surfaces. These two possibilities are not mutually exclusive. We tested for the first strategy by monitoring leeches' step frequencies on a surface of uniform roughness so that their only choice was whether to continue stepping or to stop. We tested for the second possibility by providing walking leeches with so many rough/smooth borders that they faced a choice with every step.

\section{Increased step frequency, and duration of locomotory episodes, on rougher} surfaces is consistent with a Differential Diffusion mechanism.-To test for the possibility that leeches slow down or stop on smooth substrates, we quantified the stepping behavior of individual leeches on uniform substrates across a range of textures from 80 grit to 1500 grit. Specifically, we measured the step rate (number of steps per each two-minute [Fig. 2a]) by individual leeches in unlined plastic petri dishes (representing a smooth control surface) and in dishes lined with either $80,150,320,400,600$, or 1500 grit sandpaper. Despite the fact that leeches exhibit negative responses to short-wavelength light (Jellies 2014; Bisson 2011; Harley et al 2011), step frequency was not significantly affected by the color (red vs. white) of the light in which animals were tested $(p=0.108)$. As a result, data from animals tested in either white or red light was pooled together. During the first 8 minutes, step frequencies decreased slightly on all substrate textures. But while the step frequencies for smoother substrates continued to decline, those measured on the two roughest substrates remained steady (150 grit) or even increased with time ( 80 grit). Thus by 16 minutes, the step frequencies for animals on 80 and 150 grit were clearly different from each other and from those on smoother substrates. 
For a statistical analysis of these data, we plotted the cumulative number of steps over the 30-minute test period (Fig. 2b). Consistent with the effects of grit roughness on step frequency (Fig. 2a), the total number of steps taken by animals on 80 grit sandpaper (Fig. $2 b$ ) deviated significantly from those taken on all other surfaces studied ( $p<0.01$ vs 150 grit, $p<0.001$ for all other grits, Student's t-test). The number of steps on 150 grit sandpaper also differed significantly from other conditions ( $p<0.05$ when compared against 320 and 1500 grit conditions, t-test). The cumulative step total on 80 grit sandpaper began to diverge from 150 grit after 22 minutes, from 400 grit paper after 18 minutes, from 320 and 600 grit after 16 minutes, and from from both 1500 grit and control after 12 minutes $(p<0.05$, all comparisons). There were more total steps on 150 grit sandpaper beginning at 20 minutes when compared to 1500 grit sandpaper and after 26 minutes when compared against 320 grit sandpaper $(p<0.05)$.

Thus, the behavior of leeches on uniform surfaces was consistent with the Differential Diffusion mechanism: leeches exhibited higher step frequency (Fig. 2a) and longer stepping episodes (Fig. 2b) on rougher surfaces and tended to settle on smoother surfaces.

\section{A checkerboard assay provides evidence for Smoothness Selection.-To} generate an experimental environment in which individuals faced a choice between rough and smooth substrates on every step, we placed leeches on checkerboard substrates constructed of alternating rough and smooth squares, the size of which (13 by $13 \mathrm{~mm}$ ) was chosen to approximate the length of the largest resting leech (Fig. 3a; see Materials and Methods for details).

First, we conducted five-minute trials with 100 different leeches individually on a checkerboard of the roughest and smoothest textures (80 grit and 1500 grit), starting 50 animals on a rough square and the other 50 on a smooth square. Sixteen of these 100 trials yielded no data either because of technical problems or because the leech took no steps during that trial. In the remaining 84 trials, casual inspection revealed that the leeches made more steps onto smooth squares than onto rough ones (e.g., Supplemental Video 1). Given the apparent ability of the leeches to discriminate the 80 vs 1500 grit squares, we used similarly patterned substrates to test their ability to discriminate rough from smooth textures on three intermediate pairs of substrate textures: 80 vs 150 grit, 150 vs 600 grit and 600 vs 1500 grit.

We quantified leech stepping behavior by classifying each step into one of four different categories of step transitions, rough-to-rough (RR), rough-to-smooth (RS), smooth-to-rough (SR), and smooth-to-smooth (SS), followed by statistical analyses as detailed in the Materials and Methods. As expected from casual observations (Supplemental Video 1), leeches readily discriminated between 80 grit and 1500 grit (Fig. 3b). The statistical analysis of the behavior was constrained by the fact that, for trials started by placing animals on smooth squares, the number of steps initiated from rough squares (Fig. 3b, Start Smooth, IR) was much smaller than the number of steps initiated from smooth squares (Fig. 3b, Start Smooth, IS), because of the leeches' tendency to avoid the rough surface. Note that the Start Smooth trials comparing 80 vs 1500 grit and 150 vs 600 grit averaged less than one step 
initiated from a rough square (IR) per trial (Fig. 3b). Apart from this complication, however, the differences between the observed behaviors and those predicted from a model with equally likely transitions were highly significant (Fig. 3c).

Leeches also discriminated between rough and smooth surfaces in two of the three other comparisons as well, namely, 80 vs 150 grit and 150 vs 600 grit (Fig. 3b, c). The strength of these behavioral discriminations was less pronounced than for the 80 vs 1500 comparison (as judged by their generally higher p-values), but still statistically significant for the trials with sufficient numbers of steps. In contrast, for the third comparison (600 vs 1500 grit), the step distributions generated by the best fit Monte Carlo simulations were not significantly different than those predicted by transition probabilities of 0.5 under any condition, indicating that the leeches did not discriminate between 600 and 1500 grit squares (Fig. 3b, c).

We also considered the possibility that differences in step duration might influence the behavioral outcomes in the checkerboard experiments. For example, if leeches made quicker steps from rough squares to smooth squares (RS) than from smooth squares to rough squares (SR), they would spend more time on smooth surfaces than on rough surfaces. To address this possibility we measured step duration, defined as the interval between releasing the rear sucker in successive steps. Thus, the step duration included both scanning behavior between rear sucker releases, as well as other behaviors, and complete immobility. Even for the most extreme ( 80 vs 1500 ) comparison, we found no significant difference between the average duration of RS steps $(35+/-62 \mathrm{sec} ; \mathrm{n}=76)$ and SR steps $(45+/-54 \mathrm{sec} ; \mathrm{n}=51)$.

\section{Frequency of head withdrawal during scanning correlates with substrate} texture.-A single crawl step by a leech consists of six distinct components: (1) attaching the rear sucker to the substrate near the attached front sucker, (2) release of the front sucker, (3) extending the whole body, (4) attaching the front sucker, (5) releasing the back sucker, (6) shortening the whole body to bring the rear sucker near the front sucker again. Sometimes these steps are repeated in direct succession to produce a rapid crawling behavior (Stern-Tomlinson et al. 1986). More often, however, components 3 and 4 are separated by a much more variable, elective component called "scanning". Scanning behavior consists primarily of back-and-forth sweeping motions of the front sucker and anterior midbody (Fig. 4a; Supplemental video 2), during which the ventral surface of the anterior sucker makes frequent contact with the substrate. Scanning also includes occasional "head raises", in which the animal explores above the plane (e.g., starting at roughly 4 seconds and 19 seconds in Supplemental video 2); less frequently, the scanning behavior is punctuated by rapid and pronounced retractions that lift the anterior end away from the surface and simultaneously shorten the body (Fig. 4b, c; e.g., starting at roughly 9 seconds in Supplemental video 2), a behavior that we call "head withdrawal". The speed and vigor of the head withdrawal movements gives the impression that the animal is recoiling from a noxious stimulus.

Our impression was that leeches make more head withdrawals on rough surfaces than on smooth ones. We tested this possibility by monitoring leeches crawling on uniform 
substrates of differing roughness. Specifically, we placed individual leeches onto the bottoms of plastic petri dishes covered with $80,150,320,400,600$, or 1500 grit sandpaper, or onto plastic alone as a control, smooth surface. We counted the number of head withdrawals made by 12 animals as they took 5 steps on each surface. The interstep head withdrawal frequency on 80 grit differed significantly from those on 400, 600, and 1500 grits as well as the control ( $p<0.01$ for 80 vs 400 grit, $p<0.001$ for 80 vs 600,1500, and control). Head withdrawal frequency on 150 grit significantly differed from those on all smoother conditions ( $p<0.05$ for 150 vs $320 ; p<0.01$ for 150 vs 400 ; and $p<0.001$ for 150 vs 600,1500 , and control). Both 320 and 400 grit conditions were found to be significantly different from the 1500 grit and control conditions ( $p<0.001$ for both). Leeches raised their heads more frequently on 600 grit sandpaper than on either 1500 grit sandpaper $(p<0.05)$ or the control surface $(p<0.001)$ (Fig. 4d). During head withdrawals, a leech cannot attach its front sucker to produce a step. Thus, the higher number of head withdrawals on the coarse surfaces could result in a higher probability of the anterior sucker attaching to smoother surfaces when it has access to substrates that differ in texture.

\section{DISCUSSION}

Among other reasons, Helobdella and other leeches crawl to find food (Sawyer 1981; Lent 1985; Harley etal. 2013, 2014), a mate (Sawyer 1981), other leeches (Bisson and Torre 2011), and when agitated (Willard 1981). Under the conditions of our experiments, taking leeches from their home tank and placing them in a behavioral chamber constitutes agitation. In Hirudo, agitation corresponds to the release of serotonin into the bloodstream, an effect that lasts 15-20 minutes (Willard 1981). We find that Helobdella stepping activity on uniform surfaces of different grits (Fig. 2) diminishes with a similar time constant. The sensory input that produces the increased number of head withdrawals on rougher surfaces (Fig. 4) may also cause the release of dopamine, a neuromodulator that activates crawling behavior in intact and semi-intact Hirudo (Crisp et al. 2012), and activates the crawling pattern generator in isolated Hirudo ganglia (Puhl and Mesce 2008).

The cessation of crawling or other locomotory behavior equates to settlement. The settlement preferences of diverse leech species for various artificial surfaces have previously been examined under field conditions (Adamiak-Brud et al. 2015). These studies addressed the influence of differences in substrate smoothness over only a limited range (for example smooth vs frosted glass, both of which would be perceived as smooth within our experiments). The behavioral studies presented here started with our own anecdotal observations suggesting that leeches tend to settle onto smooth surfaces, such as smooth rocks or stems of reeds, as well as on artifacts such as bottles and cans. In our first set of experiments (Fig. 1), we established that we could produce this behavior in a controlled setting, by allowing leeches to move about on a substrate divided into smoother and rougher halves lined with different grits of sandpaper. These studies showed that the leeches did move around the chamber and, if the difference in roughness was sufficiently great, they tended to settle on the smoother side. We then performed a series of more focused experiments to find possible mechanisms for this preference. 


\section{Basis for settling on smooth surfaces.}

Our behavioral data showed that leeches have two distinct mechanisms for settling on smooth surfaces: they take fewer and less frequent steps on a smooth surface than on a rough one (Fig. 2), which we call Differential Diffusion; and they select patches of smoother surface when presented with a choice of smooth and rough substrates (Fig. 3), which we call Smoothness Selection. Using these two behavioral mechanisms, a leech will move onto the smoother surface when confronted with surfaces of different textures (Smoothness Selection) and, once on a smoother surface, it would slow down (Differential Diffusion), thus spending more time on the smooth surface.

Although the two behavioral mechanisms are both driven by mechanosensation, the physiological mechanisms underlying them are likely to be quite different. The length of time for which leeches crawl and the velocity of crawling both contribute to their "diffusion rate", and we found that these parameters differ on substrates of different coarseness (Fig. 2). Thus, these data provide support for the Differential Diffusion as an explanation for the observed substrate preference behavior.

Insights into how Helobdella accomplish Smoothness Selection was provided by observing their behavior on different sandpaper grits. Helobdella did not distinguish between 600 and 1500 grits on the checkerboard (Fig. 3), and they did not distinguish between these two grits and smooth plastic on the uniform substrates (Fig. 2). From these results, we conclude that any surface between 600 grit and smooth plastic is "smooth" to a leech. They do distinguish between 150 and 600 grit and between 80 and 150 grit (Fig. 3), however, so they are able to distinguish at least 3 grades of coarseness: rough ( 80 grit), medium (150 grit), and smooth (600 grit and beyond).

The choices displayed by the leeches were never absolute: even at the most extreme differences between smooth and rough checkerboard squares (80/1500 in Fig. 3), the probability of stepping onto the preferred, smoother square was between 0.66 and 0.77 . This range of probabilities was also seen for the smooth/medium and medium/rough distinctions. This means that these animals show a 2-fold to 3 -fold preference for selecting a smoother surface, independent of the magnitude of the difference in smoothness.

The fact that the leeches could distinguish three grades of coarseness shows that they use a relative--rather than an absolute--measure of coarseness to decide where to step. This inference is clearest in considering the responses to the medium coarseness (150 grit) in the comparisons to the rough (80 grit) and to the smooth (600 grit) sandpapers (Fig. 3). When paired with the rougher surface (80/150), the 150 grit squares are prefered, i.e., it is regarded as a smooth surface. When paired with a smoother surface (150/600), however, the 150 grit is regarded as a rough surface.

\section{Mechanisms for achieving Smoothness Selection.}

How can such a relative measure of relative coarseness be accomplished? We suggest two general types of mechanisms: (1) Spatial Comparison, in which the leech simultaneously compares the textures felt at more than one body location--presumably the anterior and 
posterior suckers, because these are the only body parts that are in almost continuous contact with the substrate; and (2) Temporal Comparison, in which a leech compares the texture at a given location at different times--this mechanism could be carried out by just one body part, presumably the anterior sucker, which makes contact with the substrate during scanning movements that are a prelude to making an attachment that leads to a new step (Fig. 4). These possibilities are not mutually exclusive, so combined mechanisms are also possible.

The experiments on the rough/smooth checkerboards (Fig. 3) address the Spatial Comparison and Temporal Comparison possibilities most directly, and our data favor the Temporal Comparison mechanism. In effect, the checkerboard provides an ongoing choice between rough and smooth because the leech can reach squares with either texture at every step. Supplemental Video 1 shows that the crawling leech readily reaches the 8 adjacent squares, and in no case did a leech take a step onto the square to which the posterior sucker was attached. In both mechanisms, the anterior sucker samples possible substrates, but a Spatial Comparison mechanism simultaneously evaluates input from two locations (presumably the anterior and posterior suckers), whereas a Temporal Comparison mechanism uses input from the anterior sucker at successive times. If the leech were making a comparison between the mechanosensory input from the two suckers, the texture felt by the posterior sucker should influence the smoothness selection by the anterior sucker, but we found no evidence for such an effect; the probability of stepping onto a smooth surface is similar whether the posterior back sucker is on a smooth surface (a SS step) or a rough one (an RS step) (Fig. 3b). The fact that step duration was independent of the location of the posterior sucker also argues against the Spatial Comparison mechanism. For future work, a further test would be to have a checkerboard with three distinct surfaces, smooth, medium and rough. In such a situation, the Temporal Comparison mechanism predicts that the probability of stepping onto a square of medium texture is independent of the position of the posterior sucker, while the Spatial Comparison mechanism would predict that the probability of stepping onto a square of medium texture would be higher if the posterior sucker is on a rough square and lower if the posterior sucker is on a smooth square.

In summary, the fact that leeches move for longer times on rough surfaces than on smooth surfaces (Fig. 2a) increases the likelihood that a leech will settle on a smooth surface rather than a rough one, consistent with the Differential Diffusion mechanism. The difference between the number of steps on the coarsest and smoothest grits is highly significant--about 20 steps after 30 minutes (Fig. 2b). Each step is nearly a full extended body length, so a 20step difference could move the leech into very different territory, and in an environment with heterogeneous substrate texture, the Smoothness Selection mechanism would direct these additional steps into smoother substrates. Thus, these two mechanisms, working together, would provide both an impetus to start and stop crawling (Differential Diffusion) and an directionality that could lead to a more desirable, smoother substrate (Smoothness Selection).

Another general question is whether the sensing process itself is static (i.e., the critical mechanosensing occurs with the sucker in fixed contact with the substrate) or dynamic (i.e., the critical mechanosensing occurs as the sucker brushes the surface of the substrate). A 
priori, either static or dynamic sensing could be used for either Differential Diffusion or Smoothness Selection. For humans, dynamic processes are more effective in distinguishing texture differences of relatively smooth surfaces (600 vs 1500 grit). Unfortunately, addressing this question is beyond the scope of the present work.

\section{Possible implementation of Differential Diffusion and Smoothness Selection mechanisms.}

The Differential Diffusion mechanism suggests that the sensitivity of crawling behavior to tactile receptors does not wane, and may even increase, over time (Fig. 2). As argued above, the Smoothness Selection mechanism that is most consistent with our present results supposes that leeches react to the roughness of a surface using their anterior suckers, the most motile part of the leech. The characterization of the behavior of the anterior end of these leeches shows that they often use side-to-side scanning movements along the substrate before taking a step, and they also make occasional head withdrawals, which lift their head off the surface of the substrate (Fig. 4a; Supplemental video 2). The fact that these head withdrawals are more frequent on a rough substrate (Fig. 4b) suggests that these movements are a component of the Smoothness Selection mechanism.

The mechanosensory system of the leech provides some possibilities about how these mechanisms might be implemented. (NB: Most of this information has been garnered from work on other species than Helobdella, primarily European medicinal leeches, but mechanosensory neurons have been recorded in many other leech species (Kramer and Kuwada 1983; Kuwada and Kramer 1983; Lent 1985; Nusbaum and Kristan 1986; Baltzley et al. 2010) without finding significant differences among the species.) In each of the leech's 21 midbody segments there are 3 pairs of $T$ cells (respond to very light touch), 2 pairs of $P$ cells (respond to pressure on the skin), and 2 pairs of $\mathrm{N}$ cells (respond to strong mechanical, chemical, and thermal stimuli) (Nicholls and Baylor 1968; Carlton and McVean 1995). The same neurons are present in the head brain, which innervates the anterior sucker (Yau 1976). In general, behavioral responses require stimuli sufficient to activate $\mathrm{P}$ cells, with a boost from the activation of $\mathrm{T}$ cells; activation of $\mathrm{N}$ cells produces qualitatively different responses (Kristan 1982; Palmer et al. 2014). A reasonable hypothesis, therefore, is that the T and $\mathrm{P}$ cells are being activated as the anterior sucker is scanned over the substrate, and that occasional activation of $\mathrm{N}$ cells by the sharp edges of the grit triggers the rapid head withdrawals.

\section{Comparisons with other species}

The head movements that $H$. austinensis uses to determine a favorable substrate surface is similar to the "scanning" movements used by medicinal leeches (Hirudo verbana) to locate their prey (Harley and Wagenaar 2014). Many other animals use body extensions such as whiskers and antennae to probe their near-field environment using mechanosensors to determine whether they are about to run into an object (Harley and Ritzmann 2009), to cross over a hole in the ground (Blaesing and Cruse 2004), or even to determine what the object is (rat whisking, Bush et al., 2016). In a darkened room--especially an unfamiliar one--people use the same sorts of scanning movements with their arms and legs to avoid running into walls, doors, and furniture. 
At the level of touch sensation, humans, in describing the texture of something that they touch with their finger tips, usually mention its texture-how rough or smooth--the surface feels (Tiest 2010). People can discriminate quite fine differences between different coarseness. They can tell the difference between 600 and 1500 grit sandpapers, for instance, that differ in particle size by an average of just 10 microns (16 vs 6 microns), but only if they move their fingertips over the surface. Interestingly, for coarse sandpapers (120 vs 80 grit, which have, on average, particles of 116 vs 192 microns), people do nearly as well using static touch as they do by brushing their fingers over the surface (Hollins and Risner 2000). Similar psychophysical measurements have been made on experimental animals, using finely controlled movements of precisely machined surface textures (Eck et al. 2013). These types of studies have shown that the discriminations among smooth textures are likely made by the vibrations of the skin caused by the surfaces that move along the skin, thereby activating a particular class of tactile receptors (Pacinian corpuscles) that responds selectively to objects lightly moving over the surface of the skin (Hollins and Risner 2000; Grant et al. 2014). These responses to moving stimuli are characteristic of leech T cells (Nicholls and Baylor 1968; Lewis and Kristan 1998b; Pirschel and Kretzberg 2016), so it will be interesting to determine whether these neurons are selectively activated by the scanning movements made by leeches as they scan their environment. Because we have found that $\mathrm{T}$ and $\mathrm{P}$ neurons in Hirudo and Helobdella express many different types of orthologous transcripts (Heath-Heckman et al., in preparation), it should be possible to use genome editing approaches to selectively modify the features of specific cell types and then observe the consequences of these manipulations on the behavior of the intact animal.

\section{Supplementary Material}

Refer to Web version on PubMed Central for supplementary material.

\section{ACKNOWLEDGEMENTS}

We thank Lidia Szczupak and members of the Weisblat lab for helpful comments on the manuscript. EACHH was supported by NIH NRSA F32 NS095665.

\section{REFERENCES}

Adamiak-Brud Ż, Jabłońska-Barna I, Bielecki A, Terlecki J (2015) Settlement preferences of leeches (Clitellata: Hirudinida) for different artificial substrates. Hydrobiologia 758:275-286.

Baltzley MJ, Gaudry Q, Kristan WB Jr (2010) Species-specific behavioral patterns correlate with differences in synaptic connections between homologous mechanosensory neurons. J Comp Physiol A 196:181-97.

Belas R (2014) Biofilms, flagella, and mechanosensing of surfaces by bacteria. Trends Microbiol 22:517-527. [PubMed: 24894628]

Bisson G, Torre V (2011). Statistical characterization of social interactions and collective behavior in medicinal leeches. J Neurophysiol 106:78-90. [PubMed: 21411566]

Blackwell PG (1999) Random diffusion models for animal movement. Ecological Modelling 100:87102.

Blaesing B, Cruse H (2004) Stick insect locomotion in a complex environment: climbing over large gaps. J Exp Biol 207:1273-1286. [PubMed: 15010478] 
Bush NE, Solla SA, Hartmann MJ (2016) Whisking mechanics and active sensing. Curr Opin Neurobiol 40:178-188. [PubMed: 27632212]

Carlton T, McVean A (1995) The role of touch, pressure and nociceptive mechanoreceptors of the leech in unrestrained behavior. J Comp Physiol A 177:781-791.

Crisp KM, Gallagher BR, Mesce KA (2012) Mechanisms contributing to the dopamine induction of crawl-like bursting in leech motoneurons. J Exp Biol 215:3028-36. [PubMed: 22660774]

Eck J, Kaas AL, Mulders JLJ, Goebel R (2013) Roughness perception of unfamiliar dot patterns. Acta Psych 143:20-34.

Gaudry Q, Ruiz N, Huang T, Kristan WB 3rd, Kristan WB Jr (2010) Behavioral choice across leech species: chacun à son goût. J Exp Biol 213:1356-65. [PubMed: 20348348]

Grant RA, Itskov PM, Towal RB, Prescott TJ (2014) Active touch sensing: finger tips, whiskers, and antennae. Front Behav Neurosci 8:50. doi: 10.3389/fnbeh.2014.00050. [PubMed: 24600364]

Harley CM, English BA, Ritzmann RE (2009) Characterization of obstacle negotiation behaviors in the cockroach, Blaberus discoidalis. J Exp Biol 212:1463-1476. [PubMed: 19411540]

Harley CM, Cienfuegos J, Wagenaar DA (2011) Developmentally regulated multisensory integration for prey localization in the medicinal leech. J Exp Biol 214:3801-3807. [PubMed: 22031745]

Harley CM, Rossi M, Cienfuegos J, Wagenaar DA (2013) Discontinuous locomotion and prey sensing in the leech. J Exp Biol 216:1890-1897. [PubMed: 23785108]

Harley CM, Wagenaar DA (2014) Scanning behavior in the medicinal leech Hirudo verbana. PLoS ONE 9(1):1-7, e86120.

Hollins M, Risner SR (2000) Evidence for the duplex theory of tactile texture perception. Percep Psychophys 62:695-705.

Horne JS, Garton EO, Krone SM, Lewis JS (2007) Analyzing animal movements using Brownian bridges. Ecology 88:2354-2363. [PubMed: 17918412]

Jellies J (2014) Detection and selective avoidance of near ultraviolet radiation by an aquatic annelid: the medicinal leech. J Exp Biol 217:974-985. [PubMed: 24265432]

Kocer A (2014) Mechanisms of mechanosensing - mechanosensitive channels, function and reengineering. Curr Opin Chem Biol 29:120-127.

Kramer AP, Kuwada JY (1983) Formation of the receptive fields of leech mechanosensory neurons during embryonic development. J Neurosci 3:2474-2486. [PubMed: 6317810]

Kristan WB Jr (1982) Sensory and motor neurones responsible for the local bending response in leeches. J Exp Biol 96:161-180.

Kristan WB Jr, Calabrese RL, Friesen WO (2005) Neuronal basis of leech behaviors. Prog Neurobiol 76:279-327. [PubMed: 16260077]

Kutschera U, Langguth H, Kuo D-H, Weisblat DA (2013) Description of a new leech species from North America, Helobdella austinensis n. sp. (Hirudinea: Glossiphoniidae), with observations on its feeding behaviour. Zoosyst Evol 89: 239-246.

Kuwada JY, Kramer AP (1983) Embryonic development of the leech nervous system: Primary axon outgrowth of identified neurons. J Neurosci 3:2098-2011. [PubMed: 6619925]

Lent CM (1985) Serotonergic modulation of the feeding behavior of the medicinal leech. Br Res Bull 134:643-655.

Lewis JE, Kristan WB Jr (1998a) A neuronal network for computing population vectors in the leech. Nature 391:76-79. [PubMed: 9422507]

Lewis JE, Kristan WB Jr (1998b) Representation of touch localization by a population of leech touch sensitive neurons. J Neurophysiol 80:2584-2592. [PubMed: 9819265]

Neyman J, Pearson ES (1933) On the Problem of the Most Efficient Tests of Statistical Hypotheses. Phil Trans R Soc A: Mathematical, Physical and Engineering Sciences 231:289-337.

Nicholls JG, Baylor DA (1968) Specific modalities and receptive fields of sensory neurons in the CNS of the leech. J Neurophysiol 31:740-756. [PubMed: 5711143]

Nusbaum MP, Kristan WB Jr (1986) Swim initiation in the leech by serotonin-containing interneurons, cells 21 and 61. J Exp Biol 122:277-302. [PubMed: 3723072] 
Palmer CR, Barnett MN, Copado S, Gardezy F, Kristan WB Jr (2014) Multiplexed modulation of behavioral choice. J Exp Biol 217:2963-2973. [PubMed: 24902753]

Pastor J, Soria B, Belmonte C (1996) Properties of the nociceptive neurons of the leech segmental ganglion. J Neurophysiol 75:2268-2269. [PubMed: 8793740]

Pirschel F, Kretzberg J (2016) Multiplexed population coding of stimulus properties by leech mechanosensory cells. J Neurosci 36:3636-3647. [PubMed: 27030751]

Puhl JG, Mesce KA (2008) Dopamine activates the motor pattern for crawling in the medicinal leech. J Neurosci 28:4192-4200. [PubMed: 18417698]

Sawyer R (1981) Leech Biology and Behavior, volume 1 Oxford, Clarendon Press.

Shaw BK, Kristan WB Jr (1997) The neuronal basis of the behavioral choice between swimming and shortening in the leech: Control is not selectively exercised at higher circuit levels. J Neurosci 17:786-795. [PubMed: 8987800]

Smouse PE, Focardi S, Moorcroft PR, Kie JG, Forester JD, Morales JM (2010) Stochastic modelling of animal movement. Philos Trans R Soc Lond B Biol Sci 365:2201-2211. [PubMed: 20566497]

Stern-Tomlinson W, Nusbaum MP, Perez LE, Kristan WB Jr (1986) A kinematic study of crawling behavior in the leech, Hirudo medicinalis. J Comp Physiol 158:593-603. [PubMed: 3723440]

Tiest WM (2010) Tactual perception of material properties. Vision Res 50:2775-2782. [PubMed: 20937297]

Willard A (1981) Effects of serotonin on the generation of the motor program for swimming by the medicinal leech. J Neurosci 1:936-944. [PubMed: 7288474]

Yau KW (1976) Physiological properties and receptive fields of mechanosensory neurones in the head ganglion of the leech: comparison with homologous cells in segmental ganglia. J Physiol 263:489512. [PubMed: 1018276] 

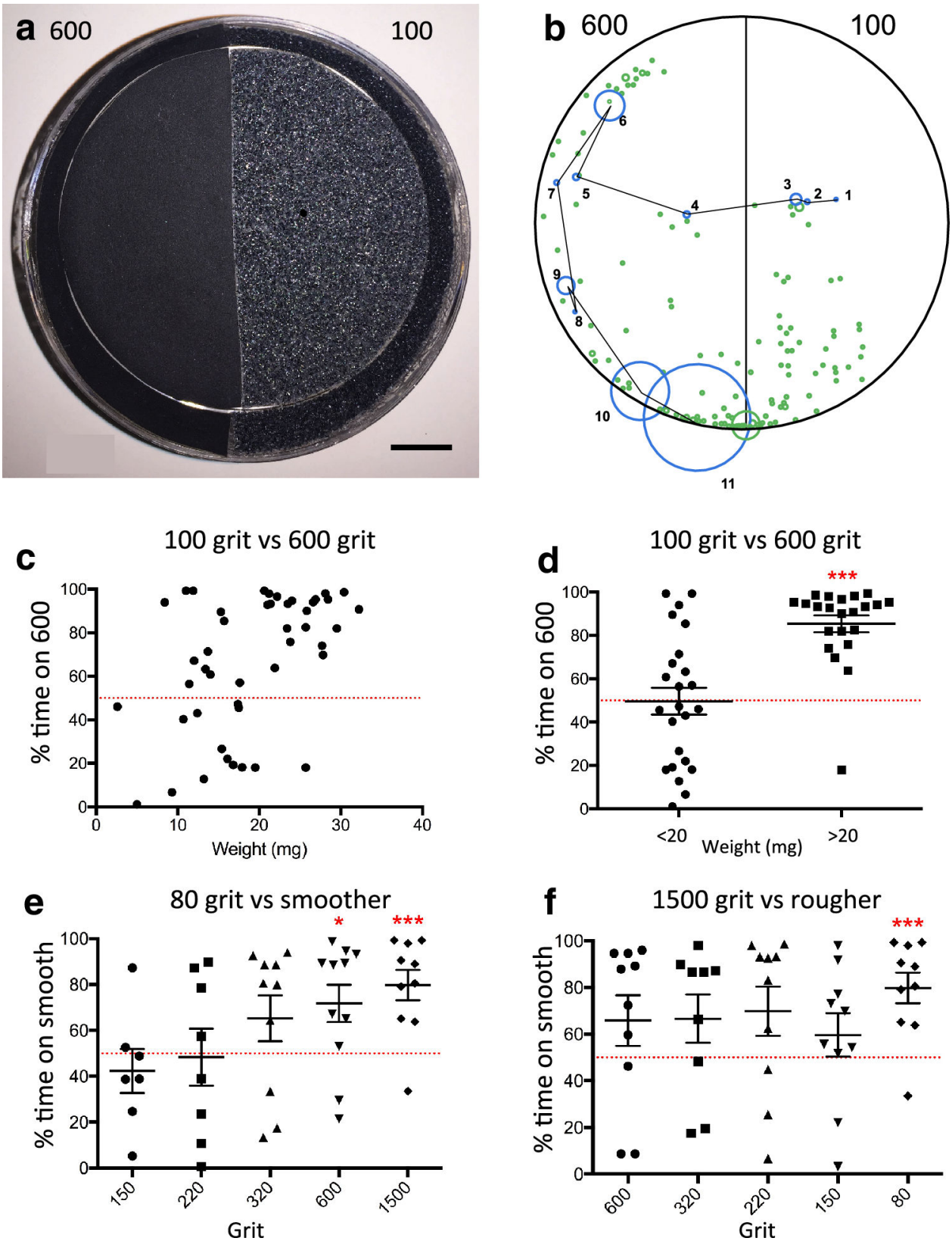

Figure 1. Heavier leeches end up preferentially on smoother substrates

(a) Representative texture preference assay chamber, photographed from above, with 600 grit and 100 grit substrates; scale bar, $1 \mathrm{~cm}$. (b) Raw data of anterior (green circles) and posterior (blue circles) sucker placement during one 25 minute trial. Circles increase in radius proportionally to time spent in one position. (c, d) Results from 46 trials in which individual leeches were placed on the rougher (100 grit) surface and allowed to navigate the apparatus freely; each point represents a single trial. In (c), the percent of the trial durations spent on the smoother substrate are plotted as a function of the weights of the 48 animals tested. In (d), the results for animals weighing less than or greater than $20 \mathrm{mg}$ are grouped separately. Leeches weighing $<20 \mathrm{mg}$ spent as much time on the rough and smooth 
sandpapers, whereas all but one of the leeches over $20 \mathrm{mg}$ spent more than $50 \%$ of time on the smoother sandpaper $(p<0.001)$. (e, f) Results from 85 similar experiments in which leeches (all greater than $20 \mathrm{mg}$ ) experienced 80 grit substrate texture on one side of the chamber and a range of smoother substrates (150, 220, 320600 and 1500 grit) on the other (e), or 1500 grit substrate texture on one side and a range of rougher substrates $(600,320$ 220,150 and 80 ) on the other (f; data for the 80 vs 1500 grit comparison are included in both plots). Most of the outlying data points represent animals that failed to cross to the smoother side until late in the trial. Error bars in d, e, f represent the average $+/-$ standard error of the mean (SEM). 

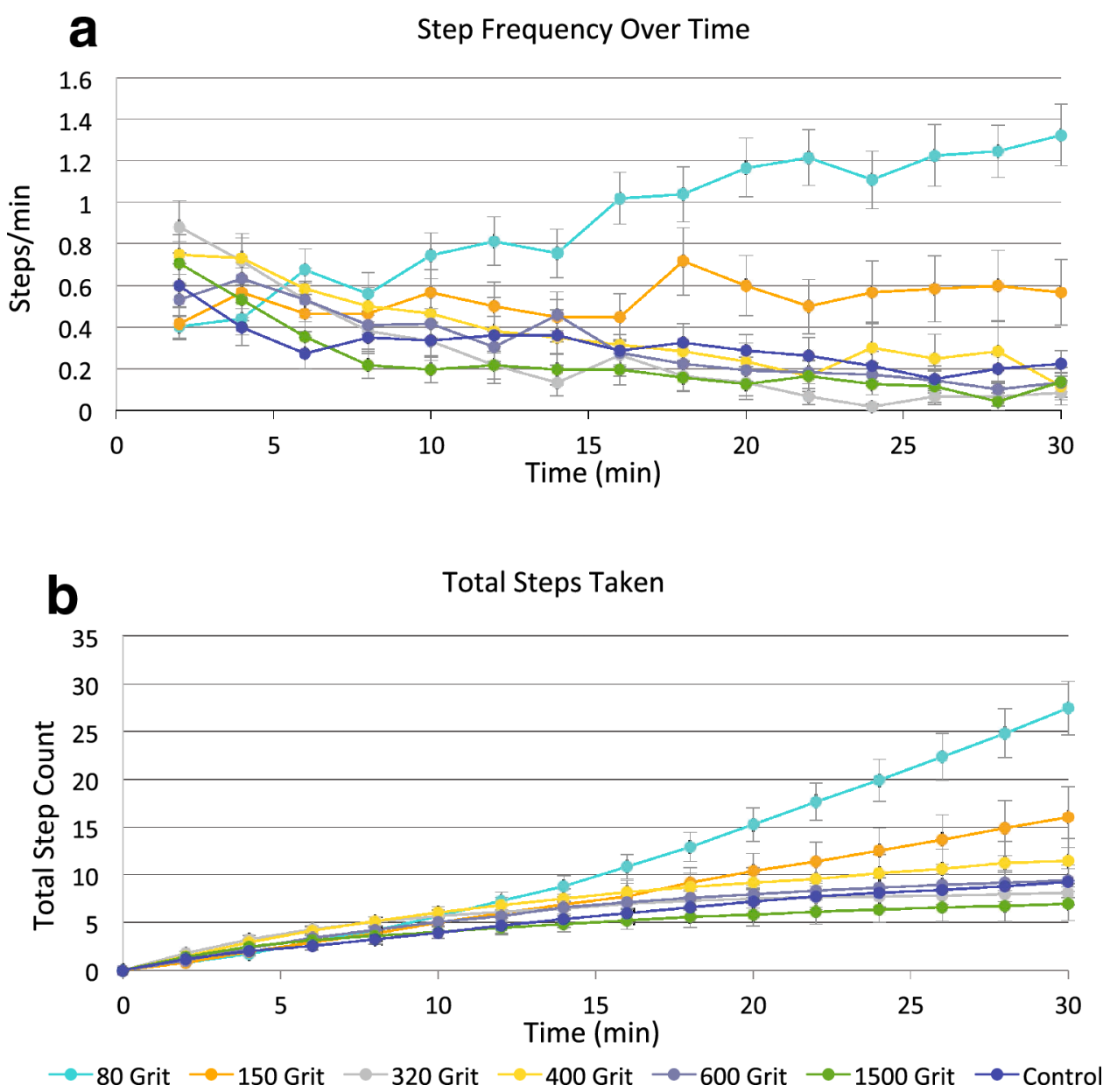

Figure 2. Step frequency and duration of locomotory episodes increased on rougher substrates. (a) Average number of steps per minute (computed from the number of steps taken during two-minute intervals) during 30 minute trials on uniform substrates covering a range of textures $(80,150,320,400,600$ and 1500 grit sandpaper, or a control smooth surface with no sandpaper). While step frequencies on the four finest grits are indistinguishable from control, decreasing over time, step frequencies remain roughly constant on 150 grit and increase over time on 80 grit (the coarsest substrate). (b) The same data as in A plotted as cumulative steps over time. The total number of steps taken over the 30 minute trial on 80 grit was significantly higher than all other conditions tested ( $p<0.01$ vs 150 grit, $p<0.001$ for all other grits, t-test). The step totals on 150 grit sandpaper also deviated from several other conditions ( $p<0.05$ vs 320 and 1500 grit sandpaper conditions, t-test). Each data point represents the average of between 29 and 51 trials. Error bars indicate SEM. 
a

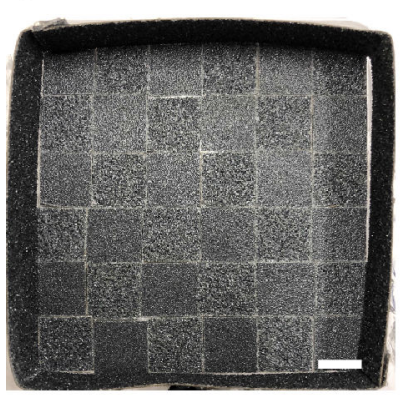

b

\begin{tabular}{c|c|c|c|c|c||cl|c|c}
\multicolumn{10}{c}{ Start Rough } \\
Comparison & $\mathrm{N}$ & $\mathrm{P}(\mathrm{SR})$ & $\mathrm{P}(\mathrm{SS})$ & \multicolumn{1}{c|}{$\mathrm{p}$-value } & $\mathrm{P}(\mathrm{RS})$ & $\mathrm{P}(\mathrm{RR})$ & \multicolumn{2}{c}{$\mathrm{p}$-value } & IR \\
\hline $80 / 1500$ & 39 & 0.34 & 0.66 & $<0.0001$ & 65 & 0.78 & 0.22 & 0.0018 & 74 \\
$150 / 600$ & 24 & 0.33 & 0.67 & 0.0005 & 41 & 0.74 & 0.26 & 0.026 & 38 \\
$80 / 150$ & 34 & 0.35 & 0.65 & 0.013 & 34 & 0.66 & 0.34 & 0.06 & 57 \\
$600 / 1500$ & 20 & 0.62 & 0.38 & 0.3 & 24 & 0.60 & 0.40 & 0.33 & 46
\end{tabular}

Start Smooth

\begin{tabular}{|c|c|c|c|c|c|c|c|c|c|}
\hline Comparison & $N$ & $P(S R)$ & $\mathrm{P}(\mathrm{SS})$ & p-value & IS & $P(R S)$ & $P(R R)$ & $\mathrm{p}$-value & IR \\
\hline $80 / 1500$ & 44 & 0.29 & 0.71 & $<0.0001$ & 128 & 0.77 & 0.23 & 0.22 & 37 \\
\hline 15 & 2 & 0.30 & 0.70 & 0.0 & 39 & 0.79 & 0.21 & 0.57 & 0 \\
\hline 8 & 25 & 0.29 & 0.71 & 0.0066 & 42 & 0.35 & 0.65 & 0.83 & 13 \\
\hline $600 / 1500$ & 24 & 0.56 & 0.44 & 0.59 & 51 & 0.58 & 0.42 & 0.76 & 29 \\
\hline
\end{tabular}

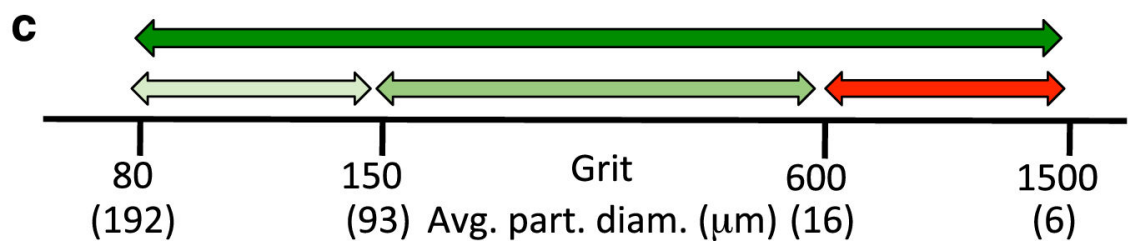

Figure 3. A checkerboard assay reveals that leeches can discriminate 80 vs 1500 grit, 80 vs 150 grit, and 150 vs 600 grit substrates.

(a) Representative checkerboard assay apparatus, showing alternating rough and smooth squares, viewed from above; scale bar, $1 \mathrm{~cm}$. (Supplemental video 1 shows leeches navigating the apparatus.) (b) Tabular summary of checkerboard experiments. For each comparison (e.g. 80 grit vs 1500 grit squares), trials were started by placing a leech on a rough square (upper table) or on a smooth square (lower table), respectively. In each row of the table, $\mathrm{N}$ is the number of usable trials (i.e., the leech took at least one step); IS and IR are the total number of steps initiated from smooth or rough squares in those trials, respectively. The number of steps per trial varied between 2.2 and 3.7 for the various conditions, with a mean of 2.9. For each set of trials, we estimated the probabilities of the four possible transitions, i.e., from smooth to rough, from smooth to smooth, from rough to smooth and from rough to rough-- $p(S R), p(S S), p(R S)$ and $p(R R)$--and tested whether the data were consistent with leeches making the four transitions randomly (see Materials and Methods for details). As a visual aid, findings with p-value $<0.05$ are highlighted in green, and those with p-value >0.05 are highlighted in red (see Materials and Methods for details). (c) Qualitative summary of the results, showing that the leeches were able to distinguish between rough and smooth surfaces in all comparisons (green arrows) except 600 grit vs 1500 grit (red arrow) and that larger differences led to stronger preferences (different shades of green). 

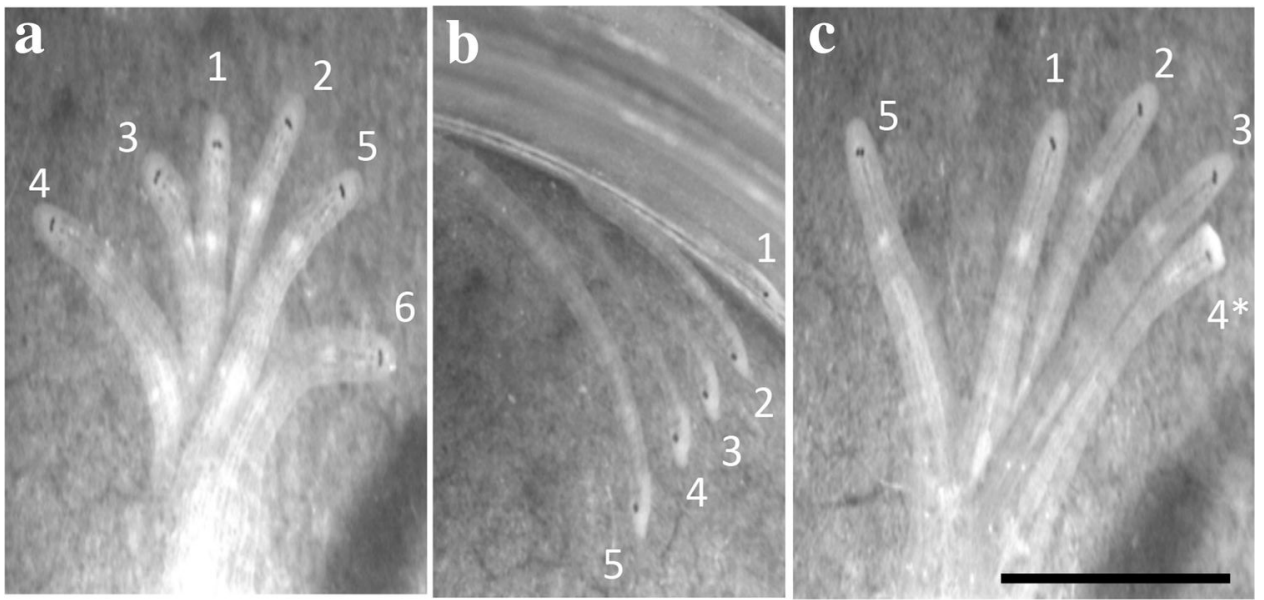

\section{d}

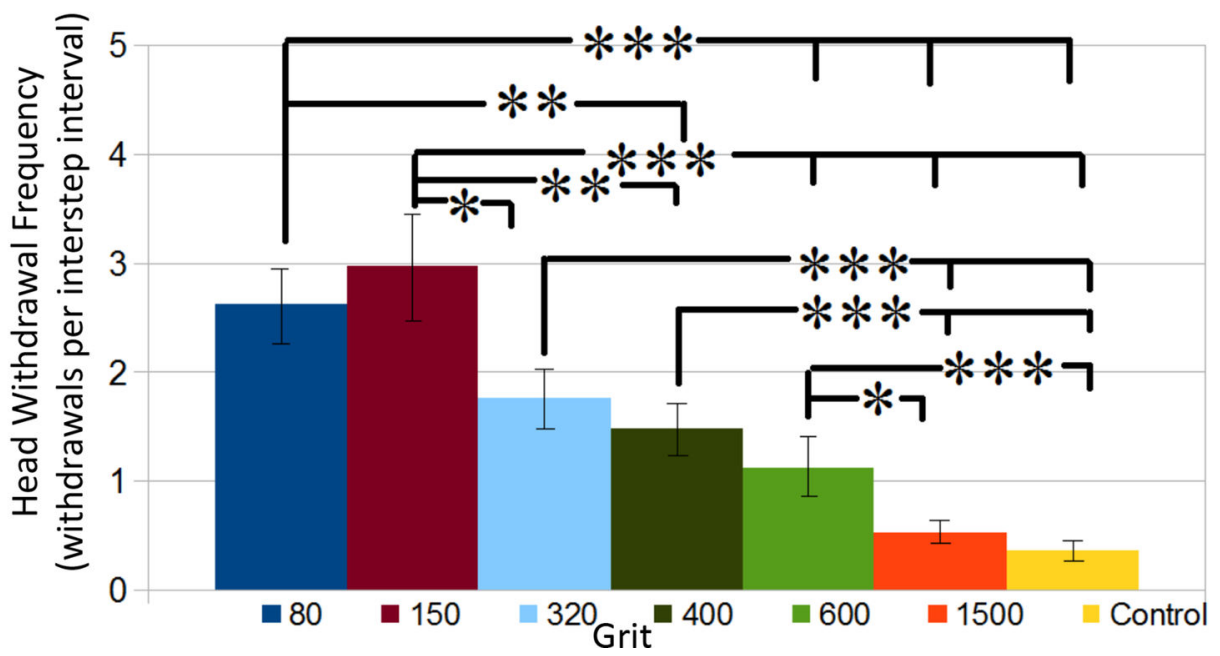

Figure 4. "Scanning", an optional exploratory component of stepping behavior, includes "sweeping", "head raising", and "head withdrawal" behaviors; head withdrawals are more frequent on rougher substrates.

Panels A-C created by merging successive images as detailed. (a) Sweeping behavior, viewed from above. Intervals between successively numbered positions were $2 \mathrm{~s}, 4 \mathrm{~s}, 10 \mathrm{~s}, 4 \mathrm{~s}$, and 4s, respectively. (b) A single episode of head raising behavior, viewed from the side, by an animal attached to the side of the petri dish. Frames 1-5 represent successive $400 \mathrm{~ms}$ time points. (c) Scanning behavior, viewed from above, with a head withdrawal (asterisk). Intervals between successively numbered positions were $1 \mathrm{~s}, 1 \mathrm{~s}, 1 \mathrm{~s}$ and $11 \mathrm{~s}$, respectively. (d) Plot of headwithdrawal averaged over 5 steps by each of 12 animals on each of 6 substrate textures and a smooth plastic control. On the diagram, $*=p<0.05$, ** $=p<0.01$, and $* * *=$ $p<0.001$, error bars indicate SEM. Scale bar, $1 \mathrm{~cm}$. 